\title{
Clinical Characteristics, Health Care Resource Utilization and Direct Medical Costs of Rotavirus Hospitalizations in Spain (2013-2018)
}

J. Arístegui

Hospital Universitario de Basurto

\section{S. Alfayate-Miguelez}

Hospital Clínico Universitario Virgen de la Arrixaca

B. Carazo-Gallego

Hospital Regional Universitario de Málaga

E. Garrote

Hospital Clínico Universitario Virgen de la Arrixaca

L. Díaz-Munilla

Complejo Hospitalario de Navarra

M. Mendizabal

Complejo Hospitalario de Navarra

E. Doménech

Hospital Germans Trias i Pujol

B. Ferrer-Lorente

Hospital Universitario y Politécnico La Fe

M. Unsaín-Mancisidor

Hospital Universitario de Donostia

\section{J. T. Ramos-Amador}

Hospital Universitario Clínico San Carlos

M. Illán-Ramos

Hospital Universitario Clínico San Carlos

B. Croche-Santander

Hospital Juan Ramón Jiménez

F. Centeno Malfaz

Hospital Universitario Río Hortega

\section{J. Rodríguez-Suárez}

Hospital Universitario Central de Asturias

\section{Cotarelo}

MSD

M. San-Martín ( $\nabla$ maria.san.martin@merck.com ) MSD

\section{Méndez-Hernández}

Hospital Germans Trias i Pujol 


\section{J. Ruiz-Contreras}

Hospital Universitario 12 de Octubre

\section{Research Article}

Keywords: Rotavirus, acute gastroenteritis, hospitalization, resources, direct costs, Spain

Posted Date: September 13th, 2021

DOI: https://doi.org/10.21203/rs.3.rs-871042/v1

License: (c) (i) This work is licensed under a Creative Commons Attribution 4.0 International License. Read Full License

Version of Record: A version of this preprint was published at Human Vaccines \&amp; Immunotherapeutics on April 18th, 2022. See the published version at https://doi.org/10.1080/21645515.2022.2046961. 


\section{Abstract}

Background: Rotavirus (RV) is the most common cause of severe gastroenteritis (GE) in infants and young children worldwide and is associated with a significant clinical and economic burden. The objective of this study was to analyze the characteristics, healthcare resource utilization and the direct medical costs related to RVGE hospitalizations.

Methods: An observational, multicenter, cross-sectional study was conducted from June 2013 to May 2018 at the pediatric departments of 12 hospitals from different Spanish regions. Children under 5 years of age admitted to the hospital with a confirmed diagnosis of RVGE were selected. Data on clinical characteristics, healthcare resource use and costs were collected from patient records and hospital databases.

Results: Most children hospitalized for RVGE did not have any previous medical condition or chronic disease. Forty-seven percent had previously visited the ER, $27 \%$ had visited a primary care pediatrician, and $15 \%$ had received pharmacological treatment prior to hospital admission due to an RVGE episode. The average length of a hospital stay for RVGE was 5.6 days, and the mean medical costs of RVGE hospitalizations per episode ranged from $3,940 €$ to $4,100 €$. The highest direct medical cost was due to the hospital stay.

Conclusion: This study shows a high burden of health resource utilization and costs related to the management of cases of RVGE requiring hospitalization. RV vaccination with high coverage rates should be considered to minimize the clinical and economic impacts of this disease on the health care system.

\section{Background}

Rotavirus (RV) remains one of the leading causes of severe diarrhea requiring hospitalization in children worldwide [1]. In Western Europe, RV is the cause of 25.3-63.5\% of all acute gastroenteritis (AGE) in children $<5$ years of age, and nosocomial RV-related acute gastroenteritis (RVGE) accounted for $47-69 \%$ of all hospitalacquired AGE leading to a prolongation of hospital stays [2, 3]. Hospitalizations due to RVGE have been associated with frequent and high utilization of healthcare resources $[3,4]$ and, as a consequence, substantial direct medical and societal costs $[4,5]$.

Two rotavirus vaccines have been available in Spain since 2006: RotaTeq ${ }^{\circledR},(\mathrm{MSD})$ [10] and Rotarix ${ }^{\circledR},(\mathrm{GSK})$ [11], but not included in the National Immunization Program. They are use under pediatricians' recommendations and fully paid by parents, reaching an intermediate vaccination coverage rate with significant differences between regions. RV vaccines have demonstrated to be highly effective, achieving $60-90 \%$ reductions in outpatient visits, emergency room (ER) visits, and hospitalizations due to severe AGE in children in many Western European and, indirectly, days missed from work of parents $[6-9,12]$.

To assess the actual burden of RVGE, both an accurate number of RVGE-related hospitalizations and their associated use of resources and related medical costs should be estimated. The use of hospital administrative databases have been found to underestimate the actual hospital admission incidence, likely due to the selected hospital discharge codification [13-15]. Furthermore, estimation of the use of medical resources and related costs might be hampered by the limited data available in those databases $[13,15,16]$. Therefore, obtaining precise information on the use of medical resources and the estimated direct medical costs related to RVGE 
hospitalizations from hospital medical records of confirmed RV patients allows for a more comprehensive evaluation of the burden of disease.

The objectives of our study were to describe the clinical characteristics and healthcare resource utilization of children under 5 years of age hospitalized due to RVGE in Spain during a 5-year period and to estimate the related direct medical costs.

\section{Methods}

\section{Study design}

This was an observational, multicenter, cross-sectional study conducted at the pediatric departments of Spanish hospitals from different regions.

Twelve hospitals from 11 different provinces that systematically tests for RV in children hospitalized due to AGE and have electronic records in the Microbiology and Pediatric Departments were selected. The analyzed data corresponded to a 5-year period (from June 2013 to May 2018).

This study was designed, conducted and reported in accordance with the Guidelines for Good Pharmacoepidemiology Practices of the International Society for Pharmacoepidemiology [17]. This study was reviewed and approved by the Euskadi Independent Ethics Committee for Research with Medicines (CEIC-E).

\section{Study population and data collection}

All children under 5 years of age admitted to the participating hospitals with a microbiologically confirmed diagnosis of RVGE during the 5-year period and with available patients' medical charts including the necessary data for study objectives analyses (clinical, diagnostic and treatment-related data) were identified as study population.

Information on the RVGE diagnosis among hospitalized children due to AGE was collected from the Microbiology Department databases. The type of test used for microbiological determination by each hospital during the study period was also collected. Hospital medical records of children included in the analysis were reviewed to collect information on their clinical characteristics and use of health care resources.

Health care resources collected comprised the utilized resources prior to hospital admission (primary care and ER visits and treatments) and the resources used during hospitalization. In the case of nosocomial RV infections only resource consumption related to the management of the RVGE were collected.

To assess direct medical costs related to health care resource utilization, the unit costs were obtained from the cost accounting of each hospital or from the Oblikue-e.Salud database, when the required information was not available from the hospital databases. Oblikue database collects Spanish unit costs obtained from more than 300 data sources retrieved from published articles, official health services tariffs of the Autonomous Communities, and discharge records from the National Health System hospitals. [18]

All study data were reviewed and recorded in an electronic case report form by the investigators.

\section{Statistical analysis}


For the description of the clinical characteristics and use of health care recourses of the RVGE hospitalizations, a sample size of 969 patients was estimated to allow the detection of a patient profile with the characteristics present in $8 \%$ of the studied population with a precision of $\pm 1.8 \%$ percentage units and a two-sided alpha risk with $p=0.05$ (type I error). Based on the Orrico-Sánchez et al. [19] study, the proportion of RVGE cases among all hospitalizations in children < 5 years of age during the postvaccine introduction period was $1.9 \%$. The study was performed in 20 hospitals from the Autonomous Community of Valencia over a 7-year period, representing approximately 20 RVGE hospitalizations per hospital and year. Therefore, by selecting a convenience sample of 12 hospitals would provide a total of 1,200 hospitalizations due to RVGE during the 5-year period with a precision of $0.79 \%$ and a level of confidence of $95 \%$. A randomized selection was performed among the total sample of children hospitalized due to RVGE in the participating hospitals.

For the clinical characteristics and resource consumption descriptive analysis, absolute and relative frequency distributions for the qualitative variables and the mean and standard deviation (SD) for quantitative variables are presented.

To calculate the cost per episode of RVGE hospitalization, the following costs were considered: ICU hospital stay, inpatient hospital stay, microbiological and other diagnostic tests, pharmacologic treatments, and hygienicdietary measures. Hospital length of stay for community-acquired RVGE episodes was calculated as follows: hospital discharge date minus the date hospital admission plus 1; and, in cases of nosocomial RVGE episodes as: date of hospital discharge minus the date of microbiological diagnosis of RVGE plus 1.

The unit costs related to the use of resources were calculated as the average unit costs obtained from the cost accounting of each hospital, or, if not available (for example, hygienic-dietary measures), it was obtained from the Oblikue-e.Salud database. Unit costs of pharmacologic treatments were obtained from the database of the General Council of the official Colleges of Pharmacists (BotPlus: https://botplusweb.portalfarma.com/). Tests/treatment for which no available unit cost was identified or for which the duration was not recorded were not included in the cost analysis.

The direct medical costs of hospitalization for RVGE were calculated as the sum of the costs of the hospital stay, diagnostic tests, pharmacological treatment and hygienic-dietary measures. Additionally, the total medical costs per RVGE episode, defined as the direct medical cost of hospitalization for RVGE and the cost derived from ER visits, out-of-hospital visits, treatment prior to hospitalizations and the length of readmissions, all related to the RVGE episode, were calculated.

The costs were estimated for each case and year during the study period. The costs were quantified by multiplying the registered natural units of the use of health care resources by the unit costs obtained for each resource. All costs were expressed in euros and corrected according to the consumer index price for 2019.

All statistical analyses were performed using the statistical program Statistical Package SAS version 9.4. A level of statistical significance of 0.05 was applied to all statistical tests.

\section{Results}

From June 2013 to May 2018, 1,731 RVGE hospitalizations in children < 5 years were collected. We randomly selected 1,089 RVGE episodes from those recorded in the hospital databases of the study hospitals, 1,002 of 
which met the selection criteria and were included for the evaluation of the clinical characteristics. Data for the health resources and direct medical costs analyses was available for 994 hospitalized children (Supplementary Fig. 1).

The mean age (SD) of cases was 13.70 (11.71) months, and 59.1\% were boys. Most children (90,1\%) and parents $(75,9 \%)$ had Spanish nationality and $70.7 \%$ of RVGE hospitalizations occurred between January and April, with a peak in March.

Among all RVGE hospitalizations, prematurity and low birth weight was reported in $13.4 \%$ and $10.1 \%$ of cases, respectively, and in $19.3 \%$ of patients, a chronic disease was informed (Table 1). Sixty percent of patients were nursing children, $26,1 \%$ of whom were breastfed (Table 1). Among other epidemiological characteristics of interest, $27.0 \%$ of patients were attending a daycare center or school at the time of admission, and $21.3 \%$ were confirmed to have household contacts with similar symptomatology, with an average of 1.34 (SD 0.74 ) contacts affected (Table 1). 
Table 1

Epidemiological characteristics of RVGE episodes hospitalized in children $<5$ years of age $(\mathrm{N}=1,002)$

\begin{tabular}{|c|c|c|}
\hline Epidemiological Characteritics & N subjects/Total sample & Percentage \\
\hline \multicolumn{3}{|l|}{ Previous conditions } \\
\hline Prematurity & $131 / 981$ & $13.4 \%$ \\
\hline$\leq 32$ weeks & $46 / 131$ & $35.4 \%$ \\
\hline$\leq 37$ weeks & $84 / 131$ & $64.6 \%$ \\
\hline Low birth weight, & $98 / 970$ & $10.1 \%$ \\
\hline$\leq 2,500 \mathrm{~g}$ & $66 / 98$ & $68.0 \%$ \\
\hline$\leq 1,500 \mathrm{~g}$ & $31 / 98$ & $32.0 \%$ \\
\hline Malabsorption syndrome & $12 / 994$ & $1.2 \%$ \\
\hline Gastroesophageal Reflux Disease & $18 / 992$ & $1.8 \%$ \\
\hline Chronic disease & $193 / 1001$ & $19.3 \%$ \\
\hline Pneumopathy & $41 / 193$ & $21.2 \%$ \\
\hline Gastrointestinal disorders & $36 / 193$ & $18.7 \%$ \\
\hline Cardiopathy & $32 / 193$ & $16.6 \%$ \\
\hline Encephalopathy & 28/193 & $14.5 \%$ \\
\hline Nephropathy & $25 / 193$ & $13.0 \%$ \\
\hline Failure to thrive & $16 / 193$ & $8.3 \%$ \\
\hline \multicolumn{3}{|l|}{ Type of feeding at hospital admission time } \\
\hline Nursing children & $456 / 750$ & $60.8 \%$ \\
\hline Breastfeeding & $119 / 456$ & $26.1 \%$ \\
\hline Formula & $253 / 456$ & $55.5 \%$ \\
\hline Mixed & $84 / 456$ & $22.8 \%$ \\
\hline Day care or school attendance & $147 / 544$ & $27.0 \%$ \\
\hline Household contacts with similar symptoms & $153 / 719$ & $21.3 \%$ \\
\hline
\end{tabular}

The most commonly reported symptoms of RVGE were diarrhea (94.4\%), vomiting (64.6\%), and fever (55.8\%), with mean (SD) durations of 2.97 (1.63), 2.16 (1.12), and 2.11 (2.25) days, respectively. The mean (SD) number of episodes per day was 7.1 (4.26) for diarrhea and 5.4 (4.48) for vomiting. Abdominal pain was reported in $11.5 \%$, and other symptoms in $21,1 \%$ of cases. 
Dehydration was the most frequent complication registered (48.5\%). The severity of dehydration is described in Fig. 2, and almost all children with dehydration $(98,8 \%)$ received intravenous rehydration at admission. The frequencies of other complications are summarized in Fig. 2. The mean number of episodes (SD) in patients presenting seizures was $2.48(2.59)$.

Among the total RVGE episodes, $16.5 \%$ were identified as nosocomial infections. Regarding hospitalization outcome, 999 patients $(99,7 \%)$ were discharged, and 3 died. Two of the deaths were reported as unrelated to the RVGE episode, and 1 was possibly related. Finally, 9 children $(0.9 \%)$ had to be readmitted following discharge due to a cause related to their previous RVGE episode.

\section{Use of resources prior to hospital admission}

Forty-six point seven percent of the children (462/990) attended the ER before the hospitalizations with a mean (SD) of $1.48(0.75)$ visits, and $27.4 \%(211 / 770)$ attended primary care pediatric office with a mean (SD) of 1.24 (0.61) visits due to the RVGE episode. In addition, $15.4 \%$ (150/973) of patients were prescribed a pharmacological treatment before their hospitalization. The frequencies of the medications and their respective treatment durations are displayed in Table 2. 
Table 2

Pharmacological and therapeutic interventions for the RVGE episodes before and during hospitalization.

\begin{tabular}{|lll|}
\hline Pharmacological tratments & N subjects (\%) & Mean Duration (Days) (SD) \\
\hline Prior to hospitalization $(\mathrm{N}=150)$ & $3(2.0 \%)$ & $2.00(1.00)$ \\
\hline Analgesics & $49(32.7 \%)$ & $2.44(1.54)$ \\
\hline Antipyretics & $2(1.3 \%)$ & - \\
\hline Antidiarrheal/antisecretory agents & $24(16.0 \%)$ & $1.68(0.75)$ \\
\hline Antiemetics & $23(15.3 \%)$ & $2.87(2.03)$ \\
\hline Ontibiotics & $71(47.3 \%)$ & $2.42(1.19)$ \\
\hline Probiotics & $6(4.0 \%)$ & $4.60(5.86)$ \\
\hline Other & $27(18.0 \%)$ & $46.13(103.10)$ \\
\hline During hospitalization $(\mathrm{N}=534)^{\star}$ & & \\
\hline
\end{tabular}

$\mathrm{N}$ in brackets: number of total evaluable subjects.; ${ }^{*} \mathrm{n}=306$ RVGE episodes with a total $\mathrm{n}=534$ pharmacologic treatments

ACE inhibitors = angiotensin-converting enzyme inhibitors; IV = intravenous; $h$ = hours; $d$ = days. 


\begin{tabular}{|c|c|c|}
\hline Pharmacological tratments & N subjects (\%) & Mean Duration (Days) (SD) \\
\hline Antibiotics & $133(24.9 \%)$ & $5.31(3.29)$ \\
\hline Antipyretics & $117(21.9 \%)$ & $1.90(1.28)$ \\
\hline Oral rehydration solutions & $89(16.7 \%)$ & $1.98(1.34)$ \\
\hline Antiemetics & $46(8.6 \%)$ & $3.03(7.55)$ \\
\hline Analgesics & $27(5.1 \%)$ & $2.44(1.46)$ \\
\hline Gastric protectors & $18(3.4 \%)$ & $9.91(12.53)$ \\
\hline Vitamins and iron supplements & $18(3.4 \%)$ & $16.78(15.78)$ \\
\hline Respiratory drugs & $16(3.0 \%)$ & $5.42(3.70)$ \\
\hline Corticosteroids & $14(2.6 \%)$ & $6.89(3.76)$ \\
\hline Anticonvulsants & $7(1.3 \%)$ & $2.00(1.73)$ \\
\hline Benzodiazepines & $6(1.1 \%)$ & $1.00(0.00)$ \\
\hline Probiotics & $5(0.9 \%)$ & $4.67(3.06)$ \\
\hline Diuretics & $4(0.7 \%)$ & $20.00()$. \\
\hline ACE inhibitors & $4(0.7 \%)$ & - \\
\hline Antihypertensive agents & $3(0.6 \%)$ & - \\
\hline Amino acids and derivatives & $3(0.6 \%)$ & $19.00()$. \\
\hline Antidiarrheal/antisecretory agents & $2(0.4 \%)$ & $10.00()$. \\
\hline Other & $22(4.1 \%)$ & $8.42(9.45)$ \\
\hline Dietary and hygiene measures during hospitalization & N subjects (\%) & Mean Duration (SD) \\
\hline IV rehydration serum $(\mathrm{N}=988)$ & 785 (79.5\%) & $33.31(34.67) \mathrm{h}$ \\
\hline IV bicarbonate $(\mathrm{N}=997)$ & $40(4.0 \%)$ & $0.59(7.02) \mathrm{h}$ \\
\hline Special diets $(N=996)$ & $56(5.6 \%)$ & $0.03(0.42) d$ \\
\hline Lactose-free milk & $25(44.6 \%)$ & $0.06(0.61) d$ \\
\hline Casein hydrolysate & $16(28.6 \%)$ & $0.01(0.11) d$ \\
\hline Whey protein hydrolysate & $4(7.1 \%)$ & $0.00(0.10) d$ \\
\hline Hydrolyzed other & $4(7.1 \%)$ & $0.03(0.39) d$ \\
\hline Parenteral nutrition & $9(16.1 \%)$ & $0.06(0.98) d$ \\
\hline Nasogastric tube feeding $(\mathrm{N}=994)$ & \multicolumn{2}{|l|}{$22(2.2 \%)$} \\
\hline
\end{tabular}




\section{Use of resources during the hospitalization}

The mean (SD) length of hospital stay due to RVGE was 5.59 (7.61) days, with a split of 5.54 (7.56) days for hospital inpatient stay and 0.05 (0.37) days in the ICU.

Immunochromatography was the most widely used test for the microbiological determination of RVGE during hospital admission (Table 3). In 94\% of the recorded RVGE episodes, other tests were performed to determine other pathogens (Table 3). Globally, 66 RVGE hospital cases tested positive for coinfection, the most frequent pathogen being adenovirus and bacteria (stool culture).

In $90.2 \%$ of the recorded RVGE episodes, other diagnostic tests were performed during hospital admission (accounting for a total of 2,138 diagnostic tests). The most frequently performed tests are summarized in Table 3. 
Table 3

Diagnostic test performed for RVGE confirmation at admission and other tests during hospitalization MICROBIOLOGICAL DIAGNOSTIC TEST $\mathrm{N}$ subjects (\%) $\quad \mathrm{N}$ of tests Mean (SD)

Test performed for microbiological diagnosis of $\operatorname{RVGE}(\mathrm{N}=1,002)$

Immunochromatography

$930(92.8 \%) \quad 0.95(0.45)$

ELISA

$1(0.1 \%)$

$0.00(0.03)$

Stool culture

$284(28.3 \%) \quad 0.30(0.52)$

PCR

$82(8.2 \%)$

$0.09(0.31)$

Other

$8(0.8 \%)$

$0.01(0.0)$

Tests for detection of coinfection with other pathogens $(\mathrm{N}=935)$

Adenovirus

Positive

\section{$858(91.8 \%)$}

28 (3.3\%)

Norovirus

$131(14.0 \%)$

Positive

Sapovirus

$2(1.5 \%)$

Positive

$54(5.8 \%)$

Coronavirus

$2(3.7 \%)$

Positive

$2(0.2 \%)$

Bocavirus

$0(0.0 \%)$

Positive

$3(0.3 \%)$

Bacteriae (stool culture)

$0(0.0 \%)$

Positive

$670(71.7 \%)$

Others

$20(3.0 \%)$

Positive

332 (35.5\%)

$14(4.2 \%)$

Other diagnostic tests $(\mathrm{N}=2,138)$ *

Blood tests

844 (39.5\%)

Complete blood count

$752(89,1 \%)$

$1.22(1.43)$

Biochemistry

$790(93,6 \%)$

$1.61(2.33)$

$\mathrm{N}$ in brackets: number of diagnostic tests performed

$\mathrm{SD}=$ Standard deviation; ELISA = Enzyme-linked immunoassay; $\mathrm{PCR}=$ Polymerase Chain Reaction; $\mathrm{CT}$ scan = Computerized tomography scan; MIR = Magnetic Resonance Imaging; ECG = Electrocardiogram; EEG = Electroencephalogram.

*A total of $n=2138$ diagnostic tests performed in 901 recorded RVGE episodes 


\begin{tabular}{|c|c|c|}
\hline MICROBIOLOGICAL DIAGNOSTIC TEST & N subjects $(\%)$ & $\mathrm{N}$ of tests Mean (SD) \\
\hline Coagulation & $47(5,6 \%)$ & $0.08(0.52)$ \\
\hline Other & $99(11,7 \%)$ & $0.25(0.64$ \\
\hline Blood gases & $673(31.5 \%)$ & $1.31(1.78)$ \\
\hline Abdominal ultrasound scan & $41(1.9 \%)$ & $0.05(0.24)$ \\
\hline Abdominal/plain X-rays & $49(2.3 \%)$ & $0.09(0.73)$ \\
\hline Ultrasound & $26(1.2 \%)$ & $0.03(0.23)$ \\
\hline CT scan & $8(0.4 \%)$ & $0.01(0.09)$ \\
\hline MRI & $2(0.1 \%)$ & $0.00(0.04)$ \\
\hline Urinalysis & $99(4.6 \%)$ & $0.12(0.39)$ \\
\hline Culture & $344(16.1 \%)$ & $0.38(0.84)$ \\
\hline ECG and EEG & $28(1.3 \%)$ & $0.03(0.17)$ \\
\hline ECG & $10(35.7 \%)$ & \\
\hline EEG & $18(64.3 \%)$ & \\
\hline Other tests & $24(1.1 \%)$ & $0.02(0.19)$ \\
\hline \multicolumn{3}{|c|}{$\mathrm{N}$ in brackets: number of diagnostic tests performed } \\
\hline \multicolumn{3}{|c|}{$\begin{array}{l}\mathrm{SD}=\text { Standard deviation; ELISA = Enzyme-linked immunoassay; } \mathrm{PCR}=\text { Polymerase Chain Reaction; } \mathrm{CT} \text { scan = } \\
\text { Computerized tomography scan; MIR = Magnetic Resonance Imaging; ECG = Electrocardiogram; EEG = } \\
\text { Electroencephalogram. }\end{array}$} \\
\hline \multicolumn{3}{|c|}{${ }^{*} A$ total of $n=2138$ diagnostic tests performed in 901 recorded RVGE episodes } \\
\hline
\end{tabular}

A total of 309 (30.9\%) children hospitalized due to RVGE received at least one pharmacological treatment during hospitalization (Table 2), mainly, antibiotics (24.9\%), antipyretics (21.9\%) and oral rehydration solutions (16.7\%). Their respective mean (SD) treatment durations were 5.31 (3.29), 1.90 (1.28), and 1.98 (1.34) days. Additionally, among the dietary and hygiene interventions during hospital admission, intravenous rehydration was administered in 785 (79.5\%) of the RVGE episodes, with an average duration of 33.31 (34.67) hours (Table 2).

\section{Direct medical costs of hospitalization for RVGE}

The average direct medical cost related to the RVGE hospitalization during the study period was $€ 3,940.38$ $(€ 5,248.85)$. The total associated cost for RVGE episodes, including the costs of medical resources reported before, during hospitalization and related to readmissions, was of $€ 4,100.11$ ( $€ 5,283.63)$ (Table 4). Among the direct medical costs, the highest was due to hospital stay on a ward with a cost of $€ 3676.63$ ( $€ 5,013)$, followed by hygienic dietary measures (€97.24; €645.81). Prior to hospitalization, ER visits represented the highest cost (Table 4). No differences were observed in the direct medical costs across the study period. Direct medical costs in each of the participating sites ranged from $€ 3,163.47(€ 3,083.06)$ to $€ 5,843.24$ ( $€ 8,716.44)$ (Supplementary Table 1). 
Table 4

Direct Medical Costs $(€)$ related to the RVGE episodes

\begin{tabular}{|c|c|c|c|c|c|c|c|}
\hline $\begin{array}{l}\text { Direct Medical } \\
\text { Costs }\end{array}$ & Global & $\begin{array}{l}2013- \\
2014\end{array}$ & $\begin{array}{l}2014- \\
2015\end{array}$ & $\begin{array}{l}2015- \\
2016\end{array}$ & $\begin{array}{l}2016- \\
2017\end{array}$ & $\begin{array}{l}2017- \\
2018\end{array}$ & $\begin{array}{l}\mathrm{p}- \\
\text { value }^{1}\end{array}$ \\
\hline \multicolumn{8}{|c|}{ During hospitalization } \\
\hline \multirow{2}{*}{$\begin{array}{l}\text { Hospital stay } \\
\text { (Ward) }\end{array}$} & $3,676.63$ & $3,949.32$ & $3,753.12$ & $3,602.46$ & $3,883.43$ & 3098.48 & \multirow[t]{2}{*}{0.7977} \\
\hline & $(5,013.00)$ & $(5,920.96)$ & $(5,341.70)$ & $(5,365.19)$ & $(5,036.95)$ & $(2550.77)$ & \\
\hline \multirow{2}{*}{$\begin{array}{l}\text { Hospital stay } \\
\text { (ICU) }\end{array}$} & 62.11 & 27.27 & 126.08 & 41.12 & 73.34 & 26.09 & \multirow[t]{2}{*}{0.4929} \\
\hline & $(498.74)$ & $(271.36)$ & (793.99) & $(315.16)$ & $(527.09)$ & $(250.77)$ & \\
\hline \multirow{2}{*}{$\begin{array}{l}\text { Microbiological } \\
\text { diagnostic test } \\
\text { Mean (SD) }\end{array}$} & 26.01 & 24.81 & 30.78 & 23.68 & 24.82 & 24.99 & \multirow[t]{2}{*}{0.6793} \\
\hline & $(45.17)$ & $(10.71)$ & (92.58) & $(11.05)$ & (11.93) & $(9.71)$ & \\
\hline \multirow{2}{*}{$\begin{array}{l}\text { Other diagnostic } \\
\text { tests } \\
\text { Mean (SD) }\end{array}$} & 76.74 & 68.68 & 80.18 & 77.46 & 73.54 & 84.51 & \multirow[t]{2}{*}{0.3027} \\
\hline & $(100.23)$ & $(82.76)$ & $(102.76)$ & $(79.43)$ & $(78.85)$ & (144.99) & \\
\hline \multirow{2}{*}{$\begin{array}{l}\text { Pharmacologic } \\
\text { treatments } \\
\text { Mean (SD) }\end{array}$} & 1.65 & 1.29 & 2.07 & 2.68 & 1.02 & 1.35 & \multirow[t]{2}{*}{0.4272} \\
\hline & $(9.58)$ & $(4.19)$ & $(6.80)$ & $(20.51)$ & $(3.65)$ & $(5.31)$ & \\
\hline \multirow{2}{*}{$\begin{array}{l}\text { Hygienic-dietary } \\
\text { measures } \\
\text { Mean (SD) }\end{array}$} & 97.24 & 36.69 & 153.64 & 121.99 & 112.04 & 52.93 & \multirow[t]{2}{*}{0.6960} \\
\hline & $(645.81)$ & $(238.63)$ & $(827.24)$ & $(965.49)$ & $(586.08)$ & $(316.76)$ & \\
\hline \multirow{2}{*}{$\begin{array}{l}\text { Total direct } \\
\text { hospitalization } \\
\text { Mean (SD) }\end{array}$} & $3,940.38$ & $4,108.07$ & $4,145.86$ & $3,869.38$ & $4,168.19$ & $3,288.36$ & \multirow[t]{2}{*}{0.8794} \\
\hline & $(5,248.85)$ & $(5,955.87)$ & $(5,921.35)$ & $(5,556.53)$ & $(5,181.77)$ & $(2,723.54)$ & \\
\hline \multicolumn{8}{|l|}{$\begin{array}{l}\text { Prior to } \\
\text { hospitalization }\end{array}$} \\
\hline \multicolumn{7}{|l|}{ Mean (SD) } & 0.9114 \\
\hline Outpatient visits & 25.98 & 25.53 & 27.99 & 18.23 & 21.95 & 35.90 & 0.0089 \\
\hline Mean (SD) & $(58.93)$ & $(60.29)$ & (64.31) & $(47.15)$ & (55.39) & (63.18) & \\
\hline
\end{tabular}

SD: Standard deviation; ER: Emergency room.

${ }^{1}$ Kruskal-Wallis test 


\begin{tabular}{|c|c|c|c|c|c|c|c|}
\hline $\begin{array}{l}\text { Direct Medical } \\
\text { Costs }\end{array}$ & Global & $\begin{array}{l}2013- \\
2014\end{array}$ & $\begin{array}{l}2014- \\
2015\end{array}$ & $\begin{array}{l}2015- \\
2016\end{array}$ & $\begin{array}{l}2016- \\
2017\end{array}$ & $\begin{array}{l}2017- \\
2018\end{array}$ & $\begin{array}{l}\text { p- } \\
\text { value }^{1}\end{array}$ \\
\hline $\begin{array}{l}\text { Prior } \\
\text { pharmacologic } \\
\text { treatments }\end{array}$ & $\begin{array}{l}0.41 \\
(1.64)\end{array}$ & $\begin{array}{l}0.51 \\
(1.85)\end{array}$ & $\begin{array}{l}0.40 \\
(1.57)\end{array}$ & $\begin{array}{l}0.56 \\
(1.92)\end{array}$ & $\begin{array}{l}0.34 \\
(1.60)\end{array}$ & $\begin{array}{l}0.29 \\
(1.20)\end{array}$ & \multirow[t]{2}{*}{0.2794} \\
\hline \multicolumn{7}{|l|}{ Mean (SD) } & \\
\hline \multicolumn{8}{|l|}{ After hospitalization } \\
\hline $\begin{array}{l}\text { Rehospitalization } \\
\text { length of stay } \\
\text { Mean (SD) }\end{array}$ & $\begin{array}{l}30.00 \\
(727.54)\end{array}$ & $\begin{array}{l}9.99 \\
(140.92)\end{array}$ & $\begin{array}{l}123.15 \\
(1,518.95)\end{array}$ & $\begin{array}{l}0.00 \\
(0.00)\end{array}$ & $\begin{array}{l}0.00 \\
(0.00)\end{array}$ & $\begin{array}{l}0.00 \\
(0.00)\end{array}$ & 0.1269 \\
\hline $\begin{array}{l}\text { Total costs } \\
\text { related with the } \\
\text { RVGE episode }\end{array}$ & $\begin{array}{l}4,100.11 \\
(5,283.63)\end{array}$ & $\begin{array}{l}4,247.80 \\
(5,932.51)\end{array}$ & $\begin{array}{l}4,399.61 \\
(6,100.49)\end{array}$ & $\begin{array}{l}3,985.23 \\
(5,536.02)\end{array}$ & $\begin{array}{l}4,293.15 \\
(5,171.30)\end{array}$ & $\begin{array}{l}3,435.39 \\
(2,708.19)\end{array}$ & 0.8323 \\
\hline \multicolumn{8}{|c|}{ SD: Standard deviation; ER: Emergency room. } \\
\hline${ }^{1}$ Kruskal-Wallis t & & & & & & & \\
\hline
\end{tabular}

\section{Discussion}

Our study showed a high burden of RVGE hospitalizations leading to significant health resource utilization and costs related to the management of these severe cases of RVGE.

Most of the hospitalized children with RVGE included in our study did not report previous medical conditions or chronic diseases [20, 21]. Moreover, among children hospitalized due to RVGE, similar to what has been observed for RVGE cases managed at primary care, attendance to daycare or school centers is not predominant [22, 23].

As described in other studies, dehydration is highly frequent among RVGE cases due to the occurrence and intensity of symptoms [24], with a not negligible proportion of cases suffering severe dehydration. However, our study was not designed to evaluate the rate of dehydration in RVGE, and the high rates of this complication could be biased, as dehydration was likely the cause of admission in many of the children. Seizures have been previously described as a complication related to RVGE. The frequency reported in our study (5\%) is within the range of what has been observed in other studies [25-27]. Although RV-related seizures are usually benign and self-limited, they may lead to ER visits and hospitalizations and considerable stress for parents [28].

Regarding the use of health care resources, we found that almost half of the patients who were hospitalized due to an RVGE episode had previously attended the ER, and approximately $30 \%$ had visited a primary care pediatrician on more than one occasion before hospital admission. Moreover, approximately $15 \%$ of all RVGE cases were hospitalized despite having received pharmacological treatment, mainly oral rehydration solutions, prior to their hospital admission. Altogether, this suggests the significant impact of RVGE episodes on the disease burden at different health care levels. 
It is important to highlight the long average length of the hospital stay (5.6 days) observed. This figure was somewhat higher than what has been reported in previous studies, ranging from 2.5 to 5 days [3, 4], but similar to those in others $[19,24]$. The results from the study performed in the Autonomous Community of Valencia including a long period of time (2002-2015), showed the relationship of the average length of hospital stay with age, with children $<2$ years having an approximately $50 \%$ longer average length of stay than the older groups, therefore, the age distributions in the different studies may explain these differences. Our study also took into consideration nosocomial infections, for whom length of hospital stay is difficult to estimate. We used the date of the microbiological diagnosis as a reference for its calculation, which may overestimate the prolongation of the stay related to RV infection in those cases. Other factors that may have contributed to longer hospital stays include the high percentage (almost $80 \%$ ) of patients in our study who needed intravenous hydration. This figure is within the upper limit of the range of many European countries [3,4].

Apart from the consequent high use of medical resources, we assume that this hospital stay must have had an important impact on the number of workdays lost by parents and caregivers, as well as other indirect costs, although the latter were not the objective of our study. In fact, it has been estimated that RVGE hospitalizations of children cause work absenteeism in nearly $70 \%$ of parents, with a mean number of days off from work of 4 , negatively impacting their quality of life [12].

Regarding pharmacological treatment and other therapeutic interventions administered during hospitalization, surprisingly, antibiotic use was reported in 25\% of the RVGE cases in our study, despite the low frequency of detected bacterial coinfection. In line with this result, a study performed in 8 European countries including Spain, assessing the appropriateness of antibiotic prescription in febrile children, showed that one-third of all antibiotic prescriptions in ED were of inappropriate or inconclusive indication [29]. This suggest that certain vaccinations, including that against RV, may contribute to an improvement in rational use of antimicrobial and to the implementation of antimicrobial stewardship guidelines.

The mean medical cost of RVGE hospitalizations per episode was 3,940€, increasing to 4,100€ when out-ofhospital costs related to the episode were considered. It is important to note that these last costs may be underestimated due to the retrospective nature of our study, using the hospital medical records of patients in which not all medical visits and treatments occurring before hospitalization may be recorded. Our study only considered the most severe presentation of disease, representing the management of cases requiring hospitalizations. Although these costs represent a significant proportion of the economic burden of disease, the clinical burden of RV disease is much higher in primary care, and the indirect costs assumed by families are not negligible. The study by Bouzón-Alejandro et al. [30] prospectively assessed indirect costs related to RVGE in Spain, showing a mean cost per case of $192.7 €$ (SD 219.8€). Therefore, additional research contributing to the global economic impact of disease estimation is warranted.

More than $95 \%$ of the average total medical cost associated with hospital admission due to an RVGE episode was attributable to the hospital stay. This was expected, as there is no specific pharmacological treatment for RV infection, and case management is focused on fluid replacement and symptom mitigation [1].

Our estimated average cost per episode is higher than that reported in other studies performed previously in Spain $[14,15,31]$. Different factors may explain these differences. First, the increase in costs over time, as there were many years of differences between the periods analyzed in those previous studies and ours. Second, the cost in those studies was mainly estimated by using the Diagnosis Related Groups for Disease (DRG). According 
to the DRG reimbursement system, patients belong to a group of diagnostically homogeneous cases; therefore, patients within the same category are similar clinically and are expected to use the same level of hospital resources. There is no specific DRG for RV, and a DRG including AGE and other miscellaneous digestive disorders in age $<18$ years was mainly used. RV is known to cause more severe AGE than other pathogens, probably leading to longer hospital stays [7]. Therefore, the lack of a specific GRD for RVGE may be responsible for an underestimation of RVGE hospitalization costs when using the DRG cost assignation. In our study, the use of different health resources related to hospitalization and unit costs calculated from hospital accounting information may have led to more precise estimations of the actual costs of RVGE-related hospitalizations. Finally, hospital length in our study seems to be longer than that obtained in other studies, as previously reported, with the cost per day of hospitalization being the major contributor to the total cost of episode management.

However, there is an important variability in the cost per RVGE hospitalization from one participating hospital to another, ranging from $€ 3,000$ to $€ 5,800$, probably due to differences in patient management protocols. Therefore, comparisons should be performed with caution.

This study has several strengths. This is the first nationwide study conducted in the post-licensure RV vaccine era evaluating the healthcare resources consumption and the direct medical costs associated to RV hospitalizations. Additionally, we included a large sample size from different hospitals and different regions and used hospital medical records as source of information, allowing us to obtain more detailed information on health care resource use and costs, unlike the use of administrative databases, which may have underestimated the actual burden of RVGE disease management at the hospital level [13, 16, 31, 32]. Furthermore, complementary information was retrieved from the microbiology department databases at each hospital.

Some study limitations must however be acknowledged. Due to its retrospective design, data availability was sometimes limited by the recorded information from study sources. A proportion of episodes in our sample were nosocomial and/or reported having previous medical conditions. It is known that RV infection may complicate the course of patients with previous conditions especially immunocompromising diseases [33]. This may have an impact on the severity and resource consumption analysis (including antibiotic use). While we cannot exclude the possibility of nosocomial transmission complicating the course of disease and resulting in cost overestimation due to RVGE, we have tried to minimize this bias by only including costs related to the treatment of RVGE in the estimations for nosocomial cases. Another limitation is the absence of an analysis of indirect costs, such as the absenteeism of parents and its economic consequences. Nonetheless, it has been confirmed that the greatest contribution to the disease burden comes from direct health care costs $[3,12]$.

\section{Conclusion}

Health care resource utilization associated with RVGE hospitalization and the derived direct costs in Spanish hospitals are high, representing a substantial economic burden of RV severe cases requiring hospital management. This fact reinforces the importance of $\mathrm{RV}$ vaccination with high vaccination coverage rates for the minimization of the clinical and economic impact of disease in the health care system. Other studies that may help to estimate the global economic burden of disease related to RV infections in Spain and the potential pharmacoeconomic impact of preventive measures are granted.

\section{Abbreviations}


AGE, acute gastroenteritis

$\mathrm{Cl}$, confidence interval

DRG, Diagnosis Related Groups for Disease

ER, emergency room

ICG, Immunochromatography

ICU, intensive care unit

RVs, Rotaviruses

GE, gastroenteritis

RVGE,

SD, standard deviation

\section{Declarations}

\section{Ethics approval and consent to participate}

The study was designed, conducted and reported in accordance with the Guidelines for Good Pharmacoepidemiology Practices of the International Society for Pharmacoepidemiology, with the ethical principles of the Declaration of Helsinki, and with the current Spanish legislation related to observational studies (Ministerial Order SAS/3470/2009).

This study was reviewed and approved by the Euskadi Independent Ethics Committee for Research with Medicines (CEIC-E).

According to the Guidelines for Good Pharmacoepidemiology Practices and the Spanish regulation for noninterventional observational studies, when using secondary data sources in which a procedure for deidentification of data is implemented, there is no need for subject informed consent collection. In the present study, only already existing data from databases and clinical records was collected, there was no interview with subjects, and all data was securely and adequately dissociated. The Euskadi Independent Ethics Committee for Research with Medicines (CEIC-E) approved this informed consent waiver.

\section{Consent for publication}

Not applicable

\section{Availability of data and materials}


The datasets used and/or analyzed during the current study are available from the corresponding author on reasonable request.

\section{Competing interest}

MC and MSM are employees of MSD Spain. All other authors do not have any competing interest.

\section{Funding}

This study was funded by MSD Spain, a subsidiary of Merck \& Co., Inc., Kenilworth, NJ, USA.

\section{Authors' Contribution}

JA, MMH, JTRA, JRC, and MSM contributed to the study design. JA, MMH, JTRA, JRC, MC and MSM participate in the analysis of data and drafted the manuscript. JA, SAM, BCG, EG, LDM, MM, ED, BFL, MUM, MIR, BCS, FCM, JRS and JRC contributed to the acquisition of data. All authors were involved in the critical review and revising of the final manuscript.

\section{Acknowledgements}

We gratefully acknowledge all site investigators of the participating hospital for their contribution to the study : Leyre Román (Hospital Universitario 12 de Octubre), Andrea Seoane (Hospital Universitario 12 de Octubre), Isabel Gimeno (Hospital Universitario 12 de Octubre), Pilar Cedena (Hospital Universitario 12 de Octubre), María Ángeles Orellana (Hospital Universitario 12 de Octubre), Bárbara Calero (Hospital Clínico Universitario Virgen de la Arrixaca), Raúl Morcillo (Hospital Clínico Universitario Virgen de la Arrixaca), Paula Vidal Lana (Hospital Regional Universitario de Málaga), Jaime Gutierrez del Álamo López (Hospital Regional Universitario de Málaga), Cristina Antúnez Fernández (Hospital Regional Universitario de Málaga), Elisa Garrote (Hospital Universitario de Basurto), Elia Doménech (Hospital Germans Trias i Pujol), Emilio Monteagudo (Hospital La Fe), Laura Pérez (Hospital La Fe), Carlos Ortí (Hospital Universitario y Politécnico La Fe), Gustavo Cilla (Hospital Universitario de Donostia), Miriam Alkorta (Hospital Universitario de Donostia), Marta Illan (Hospital Universitario Clínico San Carlos), Claudia García (Hospital Juan Ramón Jiménez), Pedro Márquez (Hospital Juan Ramón Jiménez), Mónica Frutos (Hospital Universitario Río Hortega), Carlos Alcalde (Hospital Universitario Río Hortega), Marina G. Sánchez (Hospital Universitario Río Hortega), Lucía Palacios (Hospital Universitario Río Hortega), Rosalía Cebrián (Hospital Universitario Río Hortega), Ana Isabel Elola (Hospital Universitario Central de Asturias), Alicia Pérez (Hospital Universitario Central de Asturias), María Angustias Álvarez (Hospital Universitario Central de Asturias).

Participating hospitals: Hospital Clínico Universitario Virgen de la Arrixaca (Murcia), Hospital Universitario 12 de Octubre (Madrid), Hospital Regional Universitario de Málaga (Málaga), Hospital Universitario de Basurto (Bilbao), Complejo Hospitalario de Navarra (Pamplona), Hospital Germans Trias i Pujol (Barcelona), Hospital La Fe (Valencia), Hospital Universitario de Donostia (Guipúzcoa), Hospital Universitario Clínico San Carlos (Madrid), Hospital Juan Ramón Jiménez (Huelva), Hospital Universitario Río Hortega (Valladolid), Hospital Universitario Central de Asturias (Asturias). 


\section{References}

1. Crawford SE, Ramani S, Tate JE, Parashar UD, Svensson L, Hagbom M, et al. Rotavirus infection. Nat Rev Dis Primers. 2017;3:17083.

2. Ogilvie I, Khoury H, Goetghebeur MM, El Khoury AC, Giaquinto C. Burden of community-acquired and nosocomial rotavirus gastroenteritis in the pediatric population of Western Europe: a scoping review. BMC Infect Dis. 2012;12:62.

3. Gervasi G, Capanna A, Mita V, Zaratti L, Franco E. Nosocomial rotavirus infection: an up to date evaluation of European studies. Hum Vaccin Immunother. 2016;12:2413-8.

4. Giaquinto C, van Damme P, Huet F, Gothefors L, van der Wielen M, REVEAL Study Group. Costs of community-acquired pediatric rotavirus gastroenteritis in 7 European countries: the REVEAL study. J Infect Dis. 2007;195 Suppl 1:S36-44.

5. Gleizes O, Desselberger U, Tatochenko V, Rodrigo C, Salman N, Mezner Z, et al. Nosocomial rotavirus infection in European countries: a review of the epidemiology, severity and economic burden of hospitalacquired rotavirus disease. Pediatr Infect Dis J. 2006;25:S12-21.

6. Jonesteller CL, Burnett E, Yen C, Tate JE, Parashar UD. Effectiveness of rotavirus vaccination: a systematic review of the first decade of global postlicensure data, 2006-2016. Clin Infect Dis. 2017;65:840-50.

7. Diez-Domingo J, Garces-Sanchez M, Gimenez-Sanchez F, Colomina-Rodriguez J, Martinon-Torres F. What have we learnt about rotavirus in Spain in the last 10 years? An Pediatr (Barc). 2019;91:166-79.

8. Pereira P, Vetter V, Standaert B, Benninghoff B. Fifteen years of experience with the oral live-attenuated human rotavirus vaccine: reflections on lessons learned. Expert Rev Vaccines. 2020;19:755-69.

9. de Hoog MLA, Vesikari T, Giaquinto C, Huppertz HI, Martinon-Torres F, Bruijning-Verhagen P. Report of the 5th European expert meeting on rotavirus vaccination (EEROVAC). Hum Vaccin Immunother. 2018;14:1027-34.

10. Rotateq SMPC. Summary of product characteristics. https://www.ema.europa.eu/en/documents/productinformation/rotateq-epar-product-information_en.pdf.

11. Rotarix SMPC. Summary of product characteristics. https://www.ema.europa.eu/en/documents/productinformation/rotarix-epar-product-information_en.pdf.

12. Álvarez Aldeán J, Aristegui J, López-Belmonte JL, Pedrós M, Sicilia JG. Economic and psychosocial impact of rotavirus infection in Spain: a literature review. Vaccine. 2014;32:3740-51.

13. Garcia-Basteiro AL, Bosch A, Sicuri E, Bayas JM, Trilla A, Hayes EB. Hospitalizations due to rotavirus gastroenteritis in Catalonia, Spain, 2003-2008. BMC Res Notes. 2011;4:429.

14. Gil A, Carrasco P, Jimenez R, San-Martin M, Oyaguez I, Gonzalez A. Burden of hospitalizations attributable to rotavirus infection in children in Spain, period 1999-2000. Vaccine. 2004;22:2221-5.

15. Lopez-de-Andres A, Jimenez-Garcia R, Carrasco-Garrido P, Alvaro-Meca A, Galarza PG, de Miguel AG. Hospitalizations associated with rotavirus gastroenteritis in Spain, 2001-2005. BMC Public Health. 2008;8:109.

16. Luquero FJ, Hernan Garcia C, Eiros Bouza JM, Castrodeza Sanz J, Sanchez-Padilla E, Simon Soria F, et al. Profile of paediatric admissions and emergencies during an epidemic period of rotavirus in Valladolid [Spain]. Utility of a predictive model. Gac Sanit. 2009;23:58-61. 
17. Resources. Guidelines for good pharmacoepidemiology practices (GPP). 2015. https://www.pharmacoepi.org/resources/policies/guidelines-08027/.

18. Oblikue. eSalud platform. https://www.oblikue.com/en/esalud.html.

19. Orrico-Sanchez A, Lopez-Lacort M, Perez-Vilar S, Diez-Domingo J. Long-term impact of self-financed rotavirus vaccines on rotavirus-associated hospitalizations and costs in the Valencia Region, Spain. BMC Infect Dis. 2017;17:267.

20. Dennehy PH, Cortese MM, Bégué RE, Jaeger JL, Roberts NE, Zhang R, et al. A case-control study to determine risk factors for hospitalization for Rotavirus Gastroenteritis in U.S. children. Pediatr Infect Dis J. 2006; 25:1123-1131.

21. Vesikari T, Van Damme P, Giaquinto C, Dagan R, Guarino A, Szajewska H, et al. European society for paediatric infectious diseases consensus recommendations for rotavirus vaccination in Europe: update 2014. Pediatr Infect Dis J 2015;34:635-43.

22. Aristegui J, Ferrer J, Salamanca I, Garrote E, Partidas A, San-Martin M, et al. Multicenter prospective study on the burden of rotavirus gastroenteritis in children less than 3 years of age in Spain. BMC Infect Dis 2016;16:549.

23. Gimenez-Sanchez F, Delgado-Rubio A, Martinon-Torres F, Bernaola-Iturbe E, Rotascore Research Group. Multicenter prospective study analysing the role of rotavirus on acute gastroenteritis in Spain. Acta Paediatr 2010;99:738-42.

24. García-Magán C, de Castro-López MJ, Llovo-Taboada J, Curros-Novo C, Puente-Puig M, Sánchez-Fauquier A, et al. Caracterización microbiológica de las gastroenteritis agudas virales atendidas en un servicio de pediatría en un área de alta cobertura vacunal frente a rotavirus. Enferm Infecc Microbiol Clin 2014;32:2469.

25. Kang B, Kim DH, Hong YJ, Son BK, Kim DW, Kwon YS. Comparison between febrile and afebrile seizures associated with mild rotavirus gastroenteritis. Seizure 2013;22:560-4.

26. Le Saux N, Bettinger JA, Halperin SA, Vaudry W, Scheifele DW, Canadian Immunization Monitoring Program, Active (IMPACT). Substantial morbidity for hospitalized children with community-acquired rotavirus infections: 2005-2007 IMPACT surveillance in Canadian hospitals. Pediatr Infect Dis J 2010;29:879-82.

27. Lloyd MB, Lloyd JC, Gesteland PH, Bale Jr JF. Rotavirus gastroenteritis and seizures in young children. Pediatr Neurol 2010;42:404-8.

28. Payne DC, Baggs J, Zerr DM, Klein NP, Yih K, Glanz J, et al. Protective association between rotavirus vaccination and childhood seizures in the year following vaccination in US children. Clin Infect Dis 2014;58:173-7.

29. Hagedoorn NN, Borensztajn DM, Nijman R, Balode A, von Both U, Carrol EN, et al. Variation in antibiotic prescription rates in febrile children presenting to emergency departments across Europe (MOFICHE): A muulticentre observational study. PLoS Med. 2020; 17: e1003205.

30. Bouzón-Alejandro M, Redondo-Collazo L, Sánchez-Lastres JM, Martinón-Torres N, Martinón-Sánchez JM, Martinón-Torres F, et al. Prospective evaluation of indirect costs due to acute rotavirus gastroenteritis in Spain: the ROTACOST study. BMC Pediatrics. 2011;11:81.

31. Gil-Prieto R, San Martín M, de Andrés AL, Alvaro-Meca A, González A, de Miguel AG. Hospital-acquired rotavirus infections in Spain over a ten-year period (1998-2007). Hum Vaccin. 2009;5:748-53. 
32. Gil de Miguel A, Carrasco Garrido P, Esteban Hernández J, San-Martín Rodríguez M, González López A. Ingresos hospitalarios atribuibles a rotavirus en niños de la comunidad de Madrid, período 1999-2000. An Pediatr. 2006;64:530-5.

33. Bruijning-Verhagen P, Nipshagen MD, de Graaf $H$, Bonten MJM. Rotavirus disease course among immunocompromised patients: 5-year observations froma a tertiary care medical centre. J Infect. 2017; 75:448-454.

\section{Figures}

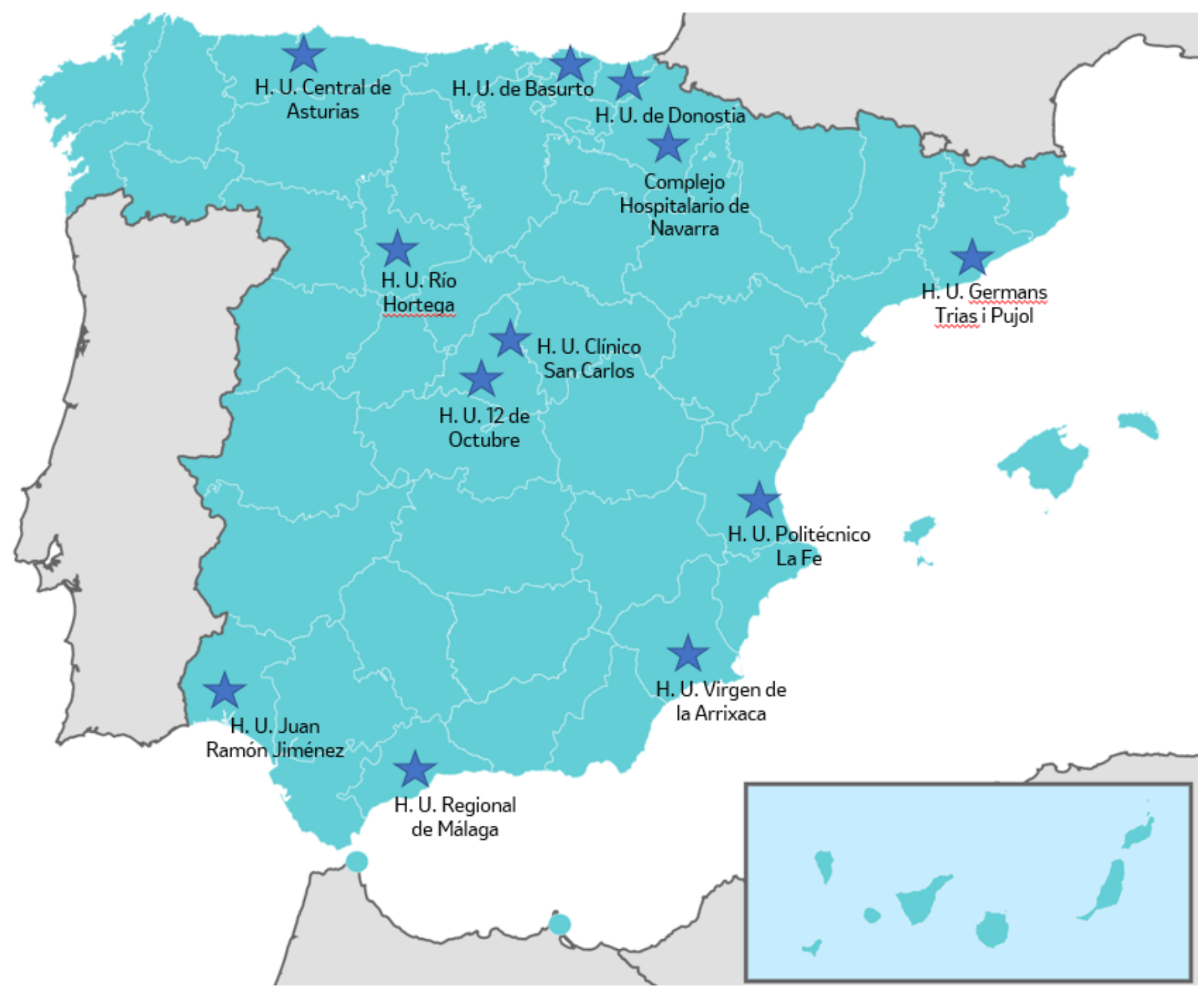

\section{Figure 1}

Geographical distribution of study hospitals 
$70 \%$

$60 \%$

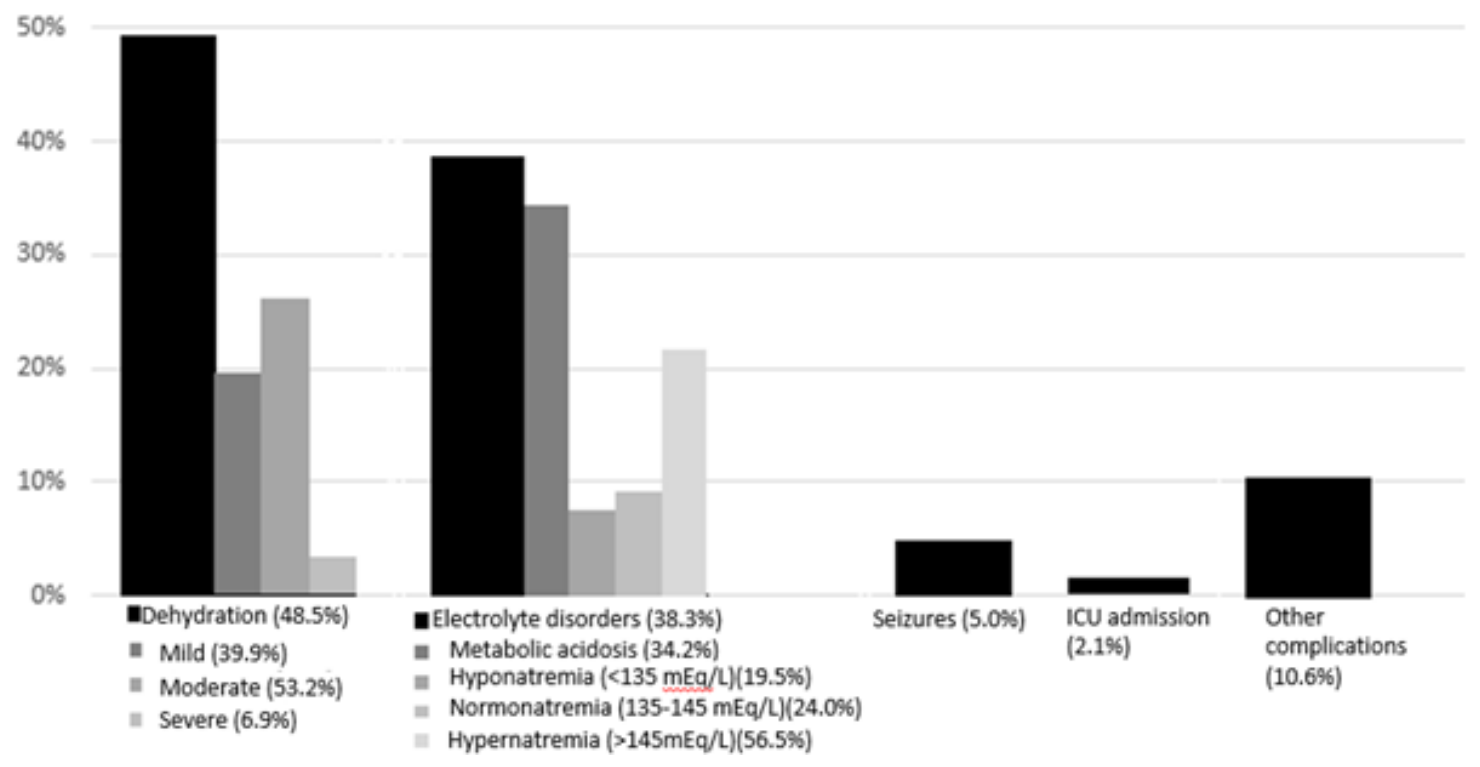

Figure 2

Frequency of complications reported in RVGE hospitalization episodes in children $<5$ years of age.

\section{Supplementary Files}

This is a list of supplementary files associated with this preprint. Click to download.

- SupplFigureFHOROSManuscriptResourcesCosts.docx

- SupplTableFHOROSManuscriptResourcesCosts.docx 